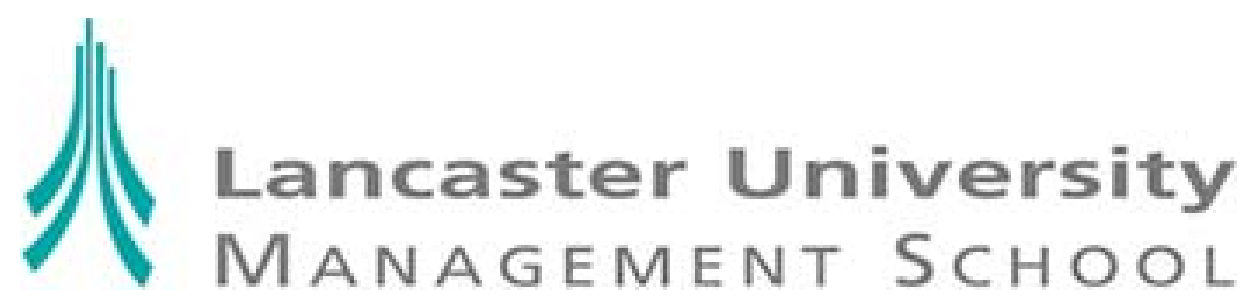

\author{
Lancaster University Management School \\ Working Paper \\ 2004/054
}

\title{
Stochastic dominance option bounds and Nth order arbitrage opportunities
}

\author{
Huang James \\ The Department of Accounting and Finance \\ Lancaster University Management School \\ Lancaster LA1 4YX \\ UK \\ CHuang James \\ All rights reserved. Short sections of text, not to exceed \\ two paragraphs, may be quoted without explicit permission, \\ provided that full acknowledgement is given.
}

The LUMS Working Papers series can be accessed at http://www/lums.co.uk/publications/ 


\title{
Stochastic Dominance Option Bounds and Nth
}

\section{Order Arbitrage Opportunities}

\author{
James Huang*
}

November 11, 2004

${ }^{*}$ Department of Accounting and Finance, Lancaster University, UK. LA1 4YX. Tel: +(44)

1524 593633, Fax: +(44) 1524 847321, Email: James.huang@lancaster.ac.uk. 


\begin{abstract}
In this paper we first derive $N$ th order stochastic dominance option bounds from concurrently expiring options. We show that these bound$\mathrm{s}$ are given by pricing kernels that have piecewise constant $(N-2)$ th derivatives. When these option bounds are violated there are $N$ th order arbitrage opportunities interpreted as (weighted average) conditional expected return comparison. We then establish a way to explore these arbitrage opportunities in option markets.
\end{abstract}

Keywords: Option bounds, Option pricing, Stochastic Dominance.

JEL Classification Numbers: G13. 


\section{Introduction}

It has been recognized that meaningful option bounds can be obtained under less strong assumptions than exact option prices. Perrakis and Ryan (1984), Ritchken (1985), and Levy (1985) derive option bounds under the assumption of risk aversion or second order stochastic dominance. ${ }^{1}$ Ritchken and Kuo (1989) derive option bounds under the assumption of higher order stochastic dominance rules. Basso and Pianca (1997) and Mathur and Ritchken (2000) obtain option pricing bounds by assuming decreasing absolute (relative) risk aversion (hereafter DARA (DRRA)).

Ryan (2003) tightens the second order stochastic dominance option bounds by using the observed price of one concurrently expiring option at a time. Huang (2004b) uses a new methodology to further improve second stochastic dominance option bounds and discusses the second order arbitrage opportunities in the markets of concurrently expiring options. The methodology is presented by Huang (2004a) which takes the advantage of options's distinctive features. Using the same methodology, Huang (2004c) improves the DARA and DRRA bounds by using the observed prices of concurrently expiring options. Huang (2004d) derives option bounds from concurrently expiring options when the pricing representative investor's relative risk aversion is bounded.

\footnotetext{
${ }^{1}$ We use SSD, TSD, and NSD to denote the second, third, and Nth order stochastic dominance.
} 
In this paper we tighten the $N$ th $(N \geq 3)$ stochastic dominance (hereafter $N \mathrm{SD}$ ) option bounds by using the observed prices of concurrently expiring options. We show that given the prices of a unit bond, underlying stock, and $n$ option prices, the $k$ th order stochastic dominance option bounds are given by a pricing kernel whose $(N-2)$ th derivative is $(n / 2)$-segmented and piecewise constant if $n$ is even or $((n+1) / 2)$-segmented and piecewise constant if $n$ is odd.

When the $N$ th stochastic dominance option bounds are violated then there are $N$ th order arbitrage opportunities. An $N$ th order arbitrage opportunity can be similarly understood to the second order arbitrage opportunity, which is interpreted by Ryan (2003) as (conditional) expected return comparison. ${ }^{2}$ An $N$ th order arbitrage opportunity can be interpreted as a weighted average conditional expected return comparison but the conditional expectation has to be taken average $N-2$ times with less weights on lower stock prices. We present the $N$ th order arbitrage portfolios which can be used to make profits when the NSD option bounds are violated.

This paper is also related to the important works by Cochrane and SaaRequejo (2000) and Bernardo and Ledoit (2000). Cochrane and Saa-Requejo derive option bounds using restrictions on the volatility of the pricing kernel, while Bernardo and Ledoit derive option pricing bounds using restrictions on the deviation of the pricing kernel from a benchmark pricing kernel.

Other related works include Lo (1987), Grundy (1991), and Constantinides

\footnotetext{
${ }^{2}$ See Huang (2004b).
} 
and Zariphopoulou (1999, 2001) who all derive option bounds under different conditions.

The structure of the remaining paper is as follows: In Section 1 we derive option bounds from concurrently expiring options when the third order stochastic dominance rule applies. In Section 2 we generalize the results in Section 1 to the case where the $N$ th $(N \geq 3)$ order stochastic dominance rule applies. In Section 3 we present the arbitrage portfolios. The final section concludes the paper.

\section{TSD Option Bounds}

In this section we derive option bounds from concurrently expiring options assuming third order stochastic dominance. According to Ritchken and Kuo (1989), applying third order stochastic dominance we have a pricing kernel that is decreasing and convex in the underlying stock price.

\subsection{Option Bound Problem and Its Dual}

Given the prices of a unit bond, a stock and some European options written on the stock with the same maturity, we want to know the bounds on the price of another option which has the same maturity when $N$ th $(N \geq 3)$ order stochastic dominance rule applies. That is,

$\max ($ or $\min ) E\left(c_{X}\left(S_{T}\right) \phi\left(S_{T}\right)\right) B_{0}$ 
subject to

$$
\begin{gathered}
\phi(x) \geq 0 ; \quad \phi^{\prime}(x) \leq 0 ; \quad \phi^{\prime}(x) \text { is increasing in } \mathrm{x} . \\
E\left(\phi\left(S_{T}\right)=1\right. \\
E\left(S_{T} \phi\left(S_{T}\right)\right) B_{0}=S_{0} \\
E\left(c_{i}\left(S_{T}\right) \phi\left(S_{T}\right)\right) B_{0}=c_{i 0}, \quad i=1, \ldots, n .
\end{gathered}
$$

The dual problem is ${ }^{3}$

$$
\min (\text { or } \max ) \alpha_{1} B_{0}+\alpha_{2} S_{0}+\sum_{i=1}^{n} \alpha_{i} c_{0}^{i}
$$

subject to

$$
\begin{aligned}
& \frac{\int_{0}^{s} E\left(\alpha_{1}+\alpha_{2} x+\sum_{i=1}^{n} \alpha_{i} c_{i}(x) \mid x<x_{0}\right) \operatorname{Pr}\left(x<x_{0}\right) d x_{0}}{\int_{0}^{S_{T}} \operatorname{Pr}\left(x<x_{0}\right) d x_{0}} \\
& \geq(\leq) \frac{\int_{0}^{S_{T}} E\left(c_{X}(x) \mid x<x_{0}\right) \operatorname{Pr}\left(x<x_{0}\right) d x_{0}}{\int_{0}^{S_{T}} \operatorname{Pr}\left(x<x_{0}\right) d x_{0}}, \text { for all } s .
\end{aligned}
$$

The above dual problem suggests a third order arbitrage opportunity if the option bound is violated. Suppose, for example, the upper bound is violated. Then by selling the over priced option $c^{X}$ and buying the arbitrage portfolio $L=\left(\alpha_{1}, \ldots, \alpha_{n+2}\right)$, we make a profit $\left(c_{0}^{X}-L_{0}>0\right)$ at time 0 . Now consider the net payoff $\left(L\left(S_{t}\right)-C_{0}^{X}\left(S_{t}\right)\right)$ at the maturity of the option. The conditional expectation of the net payoff of our position

$$
\frac{\int_{0}^{s} E\left(L\left(S_{t}\right)-C_{0}^{X}\left(S_{t}\right) \mid S_{t}<x_{0}\right) \operatorname{Pr}\left(S_{t}<x_{0}\right) d x_{0}}{\int_{0}^{S_{T}} \operatorname{Pr}\left(S_{t}<x_{0}\right) d x_{0}}
$$

will be non-negative for every $s$.

\footnotetext{
${ }^{3}$ The proof of the duality can be given by using the Ritchken and Kuo's (1989) results in the discrete case. For brevity, it is omitted.
} 
To understand third order arbitrage opportfunities we first analyze the above expression. Note is the expected net payoff under the condition that the stock price will be lower than $x_{0}$. In the case of second order arbitrage opportunity, this conditional expectation of the net payoff will be always non-negative. ${ }^{4}$ However, in the case of the third order arbitrage opportunity, this is not guaranteed to be non-negative; only a weighted average of this conditional expectation is guaranteed to be non-negative. Since for $E\left(L\left(S_{t}\right)-C_{0}^{X}\left(S_{t}\right) \mid S_{t}<x_{0}\right)$, the weight is $\operatorname{Pr}\left(S_{t}<x_{0}\right)$, the lower $x_{0}$, the less weight the conditional expectation carries. Hence the comparison of these weighted average conditional expectations is weaker the comparison of the conditional expectations. Thus the third order arbitrage opportunities are weaker than the second order arbitrage opportunities.

To solve the above option bound problem, we first solve a similar but more general problem in which we assume that not only the third order stochastic dominance rule applies but also the absolute value of the pricing kernel's first derivative is bounded from above.

We will show in this paper that under this condition, the option bounds are given by a pricing kernel that has piecewise constant first derivative, where the number of segments of the risk aversion depends on the number of observed option prices.

Moreover, we will see that for an even number of observed option prices the pricing kernel that gives the option bounds has a certain pattern while for an

\footnotetext{
${ }^{4}$ See Ryan (2003) or Huang (2004b).
} 
odd number of observed option prices the pricing kernel that gives the option bounds has a different pattern. Thus in order to explain the solutions more clearly we start with the case where we have only one observed option price then continue with the case where we have two observed options. Building on the above two cases we explore the general case where we have $n$ observed options.

\subsection{The Case with One Observed Option}

In this subsection we deal with the case where we observe the price of one concurrently expiring option. Before we proceed, we introduce two lemmas.

Lemma 1 (FSS (1999)) Assume two pricing kernels give the same stock price. If they intersect twice, then the pricing kernel with fatter tails gives higher prices of convex-payoff contingent claims writhen on the stock.

Proof: See the proof of Theorem 1 in FSS (1999).

Lemma 2 Assume two pricing kernels give the same prices of the underlying stock and an option with strike price $K$. If they intersect three times, then the pricing kernel with fatter left tail will give higher [lower] prices for all options with strike prices below [above] $K$ than the other.

Proof: See Huang (2004a).

We now derive the option bounds under the assumption that the third order stochastic dominance rule applies and the absolute value of the pricing kernel's 
first derivative is bounded from above.

Lemma 3 Assume the pricing kernel is decreasing and convex in $S_{t}$ and its first derivative is bounded below by $-\delta$. Assume the current price of a unit bond is $B_{0}$, the current price of the underlying stock is $S_{0}$ and the current price of an option with strike price $K$ is $c_{K 0}$.

- Then the upper bound for an option with strike price below $K$ is given by a pricing kernel, $\phi_{1}^{* *}(x)$, which has a three-segmented and piecewise constant first derivative. More precisely its first derivative is equal to $-\delta$ for $x<s_{1}^{* *},-\delta^{* *}$ for $x \in\left(s_{1}^{* *}, s_{2}^{* *}\right)$, and zero for $x>s_{2}^{* *}$. That is,

$$
\phi_{1}^{* *}(x)=\begin{array}{cc}
\delta^{* *}\left(s_{2}^{* *}-s_{1}^{* *}\right)+\delta\left(s_{1}^{* *}-x\right), & x<s_{1}^{* *} \\
\delta^{* *}\left(s_{2}^{* *}-x\right), & x \in\left(s_{1}^{* *}, s_{2}^{* *}\right) \\
0, & x \geq s_{2}^{* *},
\end{array}
$$

where $s_{1}^{* *}, s^{* *}$, and $\delta^{* *}$ are to be decided such that $E\left(\phi_{1}^{* *}\left(S_{t}\right)\right)=1$, $E\left(S_{t} \phi_{1}^{* *}\left(S_{t}\right)\right) / B_{0}=S_{0}$, and $E\left(c_{K}\left(S_{t}\right) \phi_{1}^{* *}\left(S_{t}\right)\right) / B_{0}=c_{K 0}$.

- The lower bound for an option with strike price below $K$ is given by a pricing kernel, $\phi_{0}^{*}(x)$, which has a two-segmented and piecewise constant first derivative. More precisely its first derivative is equal to $-\delta^{*}$ for $x<$ $s^{*}$, and zero for $x>s^{*}$, and its value is zero at its right tail. That is,

$$
\phi_{1}^{*}(x)=\left\{\begin{array}{cc}
b+\delta^{*}\left(s^{*}-x\right), & x<s^{*} \\
b, & x \geq s^{*},
\end{array}\right.
$$

where $b, \delta^{*}$, and $s^{*}$ are to be decided such that $E\left(\phi_{1}^{*}\left(S_{t}\right)\right)=1, E\left(S_{t} \phi_{0}^{*}\left(S_{t}\right)\right) / B_{0}=$ $S_{0}$, and $E\left(c_{K}\left(S_{t}\right) \phi_{1}^{*}\left(S_{t}\right)\right) / B_{0}=c_{K 0}$. 
- The upper (lower) bound for an option with strike price above $K$ is given by pricing kernel $\phi_{0}^{*}(x)\left(\phi_{0}^{* *}(x)\right)$.

Proof: From Lemma 2 we need only prove that the true pricing kernel intersects the pricing kernels that give the option bounds exactly three times and then examine which one has a fatter left tail.

We first examine $\phi_{1}^{* *}$. Note it has a three-segmented and piecewise constant first derivative. More precisely its first derivative is equal to $-\delta$ for $x<s_{1}^{* *}$, $-\delta^{* *}$ for $x \in\left(s_{1}^{* *}, s_{2}^{* *}\right)$, and zero for $x>s_{2}^{* *}$, and its value is zero at its right tail. Obviously we must have $0<\delta^{* *}<\delta$. Otherwise the true pricing kernel will intersect $\phi_{1}^{* *}$ at most twice. In this case, applying Lemma 1, we find that the two pricing kernels cannot give the same observed option price. From this, we can immediately conclude that the true pricing kernel intersects $\phi_{1}^{* *}$ exactly three times. It is not difficult to verify that $\phi_{1}^{* *}$ has fatter left tail. For $\phi_{1}^{*}$ the proof is similar. Q.E.D.

Proposition 1 Assume the pricing kernel is decreasing and convex in $S_{t}$. Assume the current price of a unit bond is $B_{0}$, the current price of the underlying stock is $S_{0}$ and the current price of an option with strike price $K$ is $c_{K 0}$.

- Then the upper bound for an option with strike price below $K$ is given by the pricing kernel $\varphi_{1}^{* *}\left(S_{t}\right)=a_{0} \frac{\delta\left(S_{t}\right)}{p\left(S_{t}\right)}+f_{1}^{* *}\left(S_{t}\right)$, where $p\left(S_{t}\right)$ is the true probability density function and $\delta\left(S_{t}\right)$ is the Dirac function and

$$
f_{1}^{* *}\left(S_{t}\right)=\left\{\begin{array}{cc}
\delta^{* *}\left(s^{* *}-x\right), & x<s^{* *} \\
0, & x \geq s^{* *},
\end{array}\right.
$$


where $a_{0}, s^{* *}$, and $\delta^{* *}$ are to be decided such that $E\left(\varphi_{1}^{* *}\left(S_{t}\right)\right)=1, E\left(S_{t} \varphi_{1}^{* *}\right.$ $\left.\left(S_{t}\right)\right) / B_{0}=S_{0}$, and $E\left(c_{K}\left(S_{t}\right) \varphi_{1}^{* *}\left(S_{t}\right)\right) / B_{0}=c_{K 0}$.

- The lower bound for an option with strike price below $K$ is given by the pricing kernel $\varphi_{1}^{*}(x)=\phi_{1}^{*}(x)$.

- The upper (lower) bound for an option with strike price above $K$ is given by pricing kernel $\phi_{0}^{*}(x)\left(\phi_{0}^{* *}(x)\right)$.

Proof: Let $\delta \rightarrow+\infty$; we immediately obtain the result from Lemma 8 .

\subsection{The Case with Two Observed Options}

In this subsection we deal with the case where we have two observed concurrently expiring options. We first introduce a lemma.

Lemma 4 Assume two pricing kernels give the same prices of the underlying stock and two options with strike prices $K_{1}$ and $K_{2}$, where $K_{1}<K_{2}$. If they intersect four times, then the pricing kernel with fatter left tail will give higher (lower) prices for options with strike prices outside (inside) $\left(K_{1}, K_{2}\right)$.

Proof: See Huang (2004a).

We now derive the option bounds under the assumption that the third order stochastic dominance rule applies and the absolute value of the pricing kernel's first derivative is bounded from above.

Lemma 5 Assume the pricing kernel is decreasing and convex in $S_{t}$ and its first derivative is bounded below by $-\delta$. Assume the current price of a unit bond 
is $B_{0}$, the current price of the underlying stock is $S_{0}$, and the current prices of two options with strike prices $K_{1}$ and $K_{2}$ are $c_{10}$ and $c_{20}$ respectively.

- Then the upper bound for an option with a strike price below $K_{1}$ or above $K_{2}$ is given by a pricing kernel, $\phi_{2}^{* *}(x)$, which has a three-segmented and piecewise constant first derivative. More precisely its first derivative is equal to $-\delta$ for $x<s_{1}^{* *},-\delta^{* *}$ for $x \in\left(s_{1}^{* *}, s_{2}^{* *}\right)$, and zero for $x>s_{2}^{* *}$, and its value is a positive constant $b$ at its right tail. That is,

$$
\phi_{2}^{* *}(x)=\left\{\begin{array}{cc}
b+\delta^{* *}\left(s_{2}^{* *}-s_{1}^{* *}\right)+\delta\left(s_{1}^{* *}-x\right), & x<s_{1}^{* *} \\
b+\delta^{* *}\left(s_{2}^{* *}-x\right), & x \in\left(s_{1}^{* *}, s_{2}^{* *}\right) \\
b, & x \geq s_{2}^{* *},
\end{array}\right.
$$

where $b, s_{1}^{* *}, s_{2}^{* *}$, and $\delta^{* *}$ are to be decided such that $E\left(\phi_{1}^{* *}\left(S_{t}\right)\right)=1$, $E\left(S_{t} \phi_{1}^{* *}\left(S_{t}\right)\right) / B_{0}=S_{0}$, and $E\left(c_{i}\left(S_{t}\right) \phi_{1}^{* *}\left(S_{t}\right)\right) / B_{0}=c_{i 0}, i=1,2$.

- The lower bound for an option with a strike price below $K_{1}$ or above $K_{2}$ is given by a pricing kernel, $\phi_{2}^{*}(x)$, which has a three-segmented and piecewise constant first derivative. More precisely its first derivative is equal to $-\delta_{1}^{*}$ for $x<s_{1}^{*},-\delta_{2}^{*}$ for $x \in\left(s_{1}^{*}, s_{2}^{*}\right)$, and zero for $x>s_{2}^{*}$. That is,

$$
\phi_{2}^{*}(x)=\left\{\begin{array}{cc}
\delta_{1}^{*}\left(s_{2}^{*}-s_{1}^{*}\right)+\delta_{2}^{*}\left(s_{1}^{*}-x\right), & x<s_{1}^{*} \\
\delta_{1}^{*}\left(s_{2}^{*}-x\right), & x \in\left(s_{1}^{*}, s_{2}^{*}\right) \\
0, & x \geq s_{2}^{*},
\end{array}\right.
$$

where $s_{1}^{*}, s_{2}^{*}, \delta_{1}^{*}$, and $\delta_{2}^{*}$ are to be decided such that $E\left(\phi_{1}^{*}\left(S_{t}\right)\right)=1$, $E\left(S_{t} \phi_{1}^{*}\left(S_{t}\right)\right) / B_{0}=S_{0}$, and $E\left(c_{i}\left(S_{t}\right) \phi_{1}^{*}\left(S_{t}\right)\right) / B_{0}=c_{i 0}, i=1,2$.

Proof: From Lemma 4 we need only prove that the true pricing kernel intersects the pricing kernels that give the option bounds exactly four times and then 
examine which one has a fatter left tail.

We first examine $\phi_{2}^{* *}$. Note it has a three-segmented and piecewise constant first derivative. More precisely its first derivative is equal to $-\delta$ for $x<s_{1}^{* *}$, $-\delta^{* *}$ for $x \in\left(s_{1}^{* *}, s_{2}^{* *}\right)$, and zero for $x>s_{2}^{* *}$, and its value is a positive constant $b$ at its right tail.

Obviously we must have $0<\delta^{* *}<\delta$ and $b>\inf _{x} \phi(x)$, where $\phi(x)$ is the true pricing kernel. Otherwise the true pricing kernel will intersect $\phi_{2}^{* *}$ at most three times. In this case, applying Lemma 2, we find that the two pricing kernels cannot give the same two observed option prices. From this, we can immediately conclude that the true pricing kernel intersects $\phi_{2}^{* *}$ exactly four times. It is not difficult to verify that $\phi_{2}^{* *}$ has fatter left tail. For $\phi_{2}^{*}$ the proof is similar. Q.E.D.

Proposition 2 Assume the pricing kernel is decreasing and convex in $S_{t}$. Assume the current price of a unit bond is $B_{0}$, the current price of the underlying stock is $S_{0}$, and the current prices of two options with strike prices $K_{1}$ and $K_{2}$ are $c_{10}$ and $c_{20}$ respectively.

- Then the upper bound for an option with a strike price below $K_{1}$ or above $K_{2}$ is given by the pricing kernel $\varphi_{2}^{* *}\left(S_{t}\right)=a_{0} \frac{\delta\left(S_{t}\right)}{p\left(S_{t}\right)}+f_{2}^{* *}\left(S_{t}\right)$, where $p\left(S_{t}\right)$ is the true probability density function and $\delta\left(S_{t}\right)$ is the Dirac function and

$$
f_{2}^{* *}\left(S_{t}\right)=\left\{\begin{array}{cc}
b+\delta^{* *}\left(s^{* *}-x\right), & x<s^{* *} \\
b, & x \geq s^{* *},
\end{array}\right.
$$

where $a_{0}, b, s^{* *}$, and $\delta^{* *}$ are to be decided such that $E\left(\varphi_{2}^{* *}\left(S_{t}\right)\right)=1$, $E\left(S_{t} \varphi_{2}^{* *}\left(S_{t}\right)\right) / B_{0}=S_{0}$, and $E\left(c_{i}\left(S_{t}\right) \varphi_{2}^{* *}\left(S_{t}\right)\right) / B_{0}=c_{i 0}, i=1,2$. 
- The lower bound for an option with a strike price below $K_{1}$ or above $K_{2}$ is given by the pricing kernel $\varphi_{2}^{*}(x)=\phi_{2}^{*}(x)$.

Proof: Let $\delta \rightarrow+\infty$; we immediately obtain the result from Lemma 9.

\subsection{The General Case}

In this subsection we deal with the case where we have $n$ observed concurrently expiring options. We first introduce a lemma.

Lemma 6 Assume two pricing kernels give the same prices of the underlying stock and options with strike prices $K_{1}, K_{2}, \ldots, K_{n}$, where $K_{1}<K_{2}<\ldots<K_{n}$. Let $K_{0}=0$ and $K_{n+1}=+\infty$. If the two pricing kernels intersect $n+2$ times then the one with fatter left tail will give higher (lower) prices for all options with strike prices between $\left(K_{2 i-2}, K_{2 i-1}\right)\left(\left(K_{2 i-1}, K_{2 i}\right)\right), i=1,2, \ldots$.

Proof: See Huang (2004a).

We now derive the option bounds under the assumption that the third order stochastic dominance rule applies and the absolute value of the pricing kernel's first derivative is bounded from above.

Lemma 7 Assume the pricing kernel is decreasing and convex in $S_{t}$ and its first derivative is bounded below by $-\delta$. Assume the current price of a unit bond is $B_{0}$, the current price of the underlying stock is $S_{0}$, and the current prices of $n$ options with strike prices $K_{1}, \ldots$, and $K_{n}$ are $c_{10}, \ldots$, and $c_{n 0}$ respectively. Let $K_{0}=0$ and $K_{n+1}=+\infty$.

- Assume $n$ is odd. Let $m=(n+1) / 2$. 
- Then the upper bound for options with strike prices between $\left(K_{2 i-2}\right.$, $\left.K_{2 i-1}\right), i=1,2, \ldots$, is given by a pricing kernel, $\phi_{n}^{* *}(x)$, which has $a(m+2)$-segmented and piecewise constant first derivative. More precisely its first derivative is equal to $-\delta$ for $x<s_{1}^{* *},-\delta_{1}^{* *}$ for $x \in\left(s_{1}^{* *}, s_{2}^{* *}\right), \ldots,-\delta_{m}^{* *}$ for $x \in\left(s_{m}^{* *}, s_{m+1}^{* *}\right)$, and zero for $x>s_{m+1}^{* *}$. That is, $\phi_{n}^{* *}(x)=$

$$
\delta_{m}^{* *}\left(s_{m+1}^{* *}-s_{m}^{* *}\right)+\ldots+\delta_{1}^{* *}\left(s_{2}^{* *}-s_{1}^{* *}\right)+\delta\left(s_{1}^{* *}-x\right), \quad x<s_{1}^{* *}
$$

$$
\begin{array}{cc}
\delta_{m}^{* *}\left(s_{m+1}^{* *}-x\right), & x \in\left(s_{m}^{* *}, s_{m+1}^{* *}\right) \\
0, & x \geq s_{m+1}^{* *},
\end{array}
$$

where $s_{1}^{* *}, \ldots, s_{m+1}^{* *}, \delta_{1}^{* *}, \ldots$, and $\delta_{m}^{* *}$ are to be decided such that $E\left(\phi_{n}^{* *}\left(S_{t}\right)\right)=1, E\left(S_{t} \phi_{n}^{* *}\left(S_{t}\right)\right) / B_{0}=S_{0}$, and $E\left(c_{i}\left(S_{t}\right) \phi_{n}^{* *}\left(S_{t}\right)\right) / B_{0}=$ $c_{i 0}, i=1,2, \ldots, n$.

- The lower bound for options with strike prices between $\left(K_{2 i-2}, K_{2 i-1}\right)$, $i=1,2, \ldots$, is given by a pricing kernel $\phi_{n}^{*}(x)$, which has a $(m+1)$ segmented and piecewise constant first derivative. More precisely its first derivative is equal to $-\delta_{1}^{*}$ for $x<s_{1}^{*}, \ldots,-\delta_{m}^{*}$ for $x \in$ $\left(s_{m-1}^{*}, s_{m}^{*}\right)$, and zero for $x>s_{m}^{*}$. That is, $\phi_{n}^{*}(x)=$

$$
\begin{array}{cc}
b+\delta_{m}^{*}\left(s_{m}^{*}-s_{m-1}^{*}\right)+\ldots+\delta_{2}^{*}\left(s_{2}^{*}-s_{1}^{*}\right)+\delta_{1}^{*}\left(s_{1}^{*}-x\right), & x<s_{1}^{*} \\
\ldots \ldots & \\
b+\delta_{m}^{*}\left(s_{m}^{*}-x\right), & x \in\left(s_{m-1}^{*}, s_{m}^{*}\right) \\
b, & x \geq s_{m}^{*},
\end{array}
$$

where $b, s_{1}^{*}, \ldots, s_{m}^{*}, \delta_{1}^{*}, \ldots$, and $\delta_{m}^{*}$ are to be decided such that 
$E\left(\phi_{n}^{*}\left(S_{t}\right)\right)=1, E\left(S_{t} \phi_{n}^{*}\left(S_{t}\right)\right) / B_{0}=S_{0}$, and $E\left(c_{i}\left(S_{t}\right) \phi_{n}^{*}\left(S_{t}\right)\right) / B_{0}=$ $c_{i 0}, i=1,2, \ldots, n$.

- Assume $n$ is even. Let $m=n / 2$.

- Then the upper bound for options with strike prices between $\left(K_{2 i-2}\right.$, $\left.K_{2 i-1}\right), i=1,2, \ldots$, is given by a pricing kernel, $\phi_{n}^{* *}(x)$, which has $a(m+2)$-segmented and piecewise constant first derivative. More precisely its first derivative is equal to $-\delta$ for $x<s_{1}^{* *},-\delta_{1}^{* *}$ for $x \in\left(s_{1}^{* *}, s_{2}^{* *}\right), \ldots,-\delta_{m}^{* *}$ for $x \in\left(s_{m}^{* *}, s_{m+1}^{* *}\right)$, and zero for $x>s_{m+1}^{* *}$, and its value at its right tail is a positive constant $b$. That is, $\phi_{n}^{* *}(x)=$ $b+\delta_{m}^{* *}\left(s_{m+1}^{* *}-s_{m}^{* *}\right)+\ldots+\delta_{1}^{* *}\left(s_{2}^{* *}-s_{1}^{* *}\right)+\delta\left(s_{1}^{* *}-x\right), \quad x<s_{1}^{* *}$

$$
\begin{array}{cc}
b+\delta_{m}^{* *}\left(s_{m+1}^{* *}-x\right), & x \in\left(s_{m}^{* *}, s_{m+1}^{* *}\right) \\
b, & x \geq s_{m+1}^{* *},
\end{array}
$$

where $b, s_{1}^{* *}, \ldots, s_{m+1}^{* *}, \delta_{1}^{* *}, \ldots$, and $\delta_{m}^{* *}$ are to be decided such that $E\left(\phi_{n}^{* *}\left(S_{t}\right)\right)=1, E\left(S_{t} \phi_{n}^{* *}\left(S_{t}\right)\right) / B_{0}=S_{0}$, and $E\left(c_{i}\left(S_{t}\right) \phi_{n}^{* *}\left(S_{t}\right)\right) / B_{0}=$ $c_{i 0}, i=1,2, \ldots, n$.

- The lower bound for options with strike prices between $\left(K_{2 i-2}, K_{2 i-1}\right)$, $i=1,2, \ldots$, is given by a pricing kernel, $\phi_{n}^{*}(x)$, which has a $(m+2)$ segmented and piecewise constant first derivative. More precisely it$s$ first derivative is equal to $-\delta_{1}^{*}$ for $x<s_{1}^{*}, \ldots,-\delta_{m+1}^{*}$ for $x \in$ 


$$
\begin{array}{cc}
\left(s_{m}^{*}, s_{m+1}^{*}\right), \text { and zero for } x>s_{m+1}^{*} . & \\
\delta_{m+1}^{*}\left(s_{m+1}^{*}-s_{m}^{*}\right)+\ldots+\delta_{2}^{*}\left(s_{2}^{*}-s_{1}^{*}\right)+\delta_{1}^{*}\left(s_{1}^{*}-x\right), & x<s_{1}^{*} \\
\ldots \ldots & \\
\delta_{m+1}^{*}\left(s_{m+1}^{*}-x\right), & x \in\left(s_{m-1}^{*}, s_{m}^{*}\right) \\
0, & x \geq s_{m+1}^{*},
\end{array}
$$

where $s_{1}^{*}, \ldots, s_{m+1}^{*}, \delta_{1}^{*}, \ldots$, and $\delta_{m+1}^{*}$ are to be decided such that $E\left(\phi_{n}^{*}\left(S_{t}\right)\right)=1, E\left(S_{t} \phi_{n}^{*}\left(S_{t}\right)\right) / B_{0}=S_{0}$, and $E\left(c_{i}\left(S_{t}\right) \phi_{n}^{*}\left(S_{t}\right)\right) / B_{0}=$ $c_{i 0}, i=1,2, \ldots, n$.

Proof: From Lemma 6 we need only prove that the true pricing kernel intersects the pricing kernels that give the option bounds exactly $(n+2)$ times and then examine which one has a fatter left tail.

We first examine $\phi_{n}^{* *}$. Assume $n$ is odd. Note it has a $(\mathrm{m}+2)$-segmented and piecewise constant first derivative, where $m=(n+1) / 2$. More precisely, its first derivative is equal to $-\delta$ for $x<s_{1}^{* *},-\delta_{1}^{* *}$ for $x \in\left(s_{1}^{* *}, s_{2}^{* *}\right), \ldots,-\delta_{m}^{* *}$ for $x \in\left(s_{m}^{* *}, s_{m+1}^{* *}\right)$, and zero for $x>s_{m+1}^{* *}$, and its value at its right tail is 0 .

Obviously we must have $\delta>\delta_{1}^{* *}>\ldots \delta_{m}^{* *}>0$ and $b>\inf _{x} \phi(x)$, where $\phi(x)$ is the true pricing kernel. Otherwise the true pricing kernel will intersect $\phi_{n}^{* *}$ at most $n+1$ times. In this case, applying Lemma 6 , we find that the two pricing kernels cannot give the same $n$ observed option prices. From this, we can immediately conclude that the true pricing kernel intersects $\phi_{n}^{* *}$ exactly $n+2$ times. It is not difficult to verify that $\phi_{n}^{* *}$ has fatter left tail.

Assume $n$ is even. Note it has a $(\mathrm{m}+2)$-segmented and piecewise constant first derivative, where $m=n / 2$. More precisely, its first derivative is equal to 
$-\delta$ for $x<s_{1}^{* *},-\delta_{1}^{* *}$ for $x \in\left(s_{1}^{* *}, s_{2}^{* *}\right), \ldots,-\delta_{m}^{* *}$ for $x \in\left(s_{m}^{* *}, s_{m+1}^{* *}\right)$, and zero for $x>s_{m+1}^{* *}$, and its value at its right tail is a positive constant $b$.

Obviously we must have $\delta>\delta_{1}^{* *}>\ldots \delta_{m}^{* *}>0$ and $b>\inf _{x} \phi(x)$, where $\phi(x)$ is the true pricing kernel. Otherwise the true pricing kernel will intersect $\phi_{n}^{* *}$ at most $n+1$ times. In this case, applying Lemma 6 , we find that the two pricing kernels cannot give the same $n$ observed option prices. From this, we can immediately conclude that the true pricing kernel intersects $\phi_{n}^{* *}$ exactly $n+2$ times. It is not difficult to verify that $\phi_{n}^{* *}$ has fatter left tail.

For $\phi_{n}^{*}$ the proof is similar. Q.E.D.

Proposition 3 Assume the pricing kernel is decreasing and convex in $S_{t}$. Assume the current price of a unit bond is $B_{0}$, the current price of the underlying stock is $S_{0}$, and the current prices of $n$ options with strike prices $K_{1}, \ldots$, and $K_{n}$ are $c_{10}, \ldots$, and $c_{n 0}$ respectively. Let $K_{0}=0$ and $K_{n+1}=+\infty$.

- Assume $n$ is odd. Let $m=(n+1) / 2$.

- Then the upper bound for options with strike prices between $\left(K_{2 i-2}\right.$, $\left.K_{2 i-1}\right), i=1,2, \ldots$, is given by the pricing kernel $\varphi_{n}^{* *}\left(S_{t}\right)=a_{0} \frac{\delta\left(S_{t}\right)}{p\left(S_{t}\right)}+$ $f_{n}^{* *}\left(S_{t}\right)$, where $p\left(S_{t}\right)$ is the true probability density function, $\delta\left(S_{t}\right)$ is the Dirac function, and $f_{n}^{* *}(x)=$

$$
\begin{array}{cc}
\delta_{m}^{* *}\left(s_{m+1}^{* *}-s_{m}^{* *}\right)+\ldots+\delta_{2}^{* *}\left(s_{3}^{* *}-s_{2}^{* *}\right)+\delta_{1}^{* *}\left(s_{2}^{* *}-x\right), & x<s_{2}^{* *} \\
\ldots \ldots & \\
\delta_{m}^{* *}\left(s_{m+1}^{* *}-x\right), & x \in\left(s_{m}^{* *}, s_{m+1}^{* *}\right) \\
0, & x \geq s_{m+1}^{* *},
\end{array}
$$


where $a_{0}, s_{2}^{* *}, \ldots, s_{m+1}^{* *}, \delta_{1}^{* *}, \ldots$, and $\delta_{m}^{* *}$ are to be decided such that $E\left(\phi_{n}^{* *}\left(S_{t}\right)\right)=1, E\left(S_{t} \phi_{n}^{* *}\left(S_{t}\right)\right) / B_{0}=S_{0}$, and $E\left(c_{i}\left(S_{t}\right) \phi_{n}^{* *}\left(S_{t}\right)\right) / B_{0}=$ $c_{i 0}, i=1,2, \ldots, n$.

- The lower bound for options with strike prices between $\left(K_{2 i-2}, K_{2 i-1}\right)$, $i=1,2, \ldots$, is given by the pricing kernel $\varphi_{n}^{*}(x)=$

$$
\begin{array}{cc}
b+\delta_{m}^{*}\left(s_{m}^{*}-s_{m-1}^{*}\right)+\ldots+\delta_{2}^{*}\left(s_{2}^{*}-s_{1}^{*}\right)+\delta_{1}^{*}\left(s_{1}^{*}-x\right), & x<s_{1}^{*} \\
\cdots \ldots & x \in\left(s_{m-1}^{*}, s_{m}^{*}\right) \\
b+\delta_{m}^{*}\left(s_{m}^{*}-x\right), & x \geq s_{m}^{*}, \\
b, &
\end{array}
$$

where $b, s_{1}^{*}, \ldots, s_{m}^{*}, \delta_{1}^{*}, \ldots$, and $\delta_{m}^{*}$ are to be decided such that $E\left(\phi_{n}^{*}\left(S_{t}\right)\right)=1, E\left(S_{t} \phi_{n}^{*}\left(S_{t}\right)\right) / B_{0}=S_{0}$, and $E\left(c_{i}\left(S_{t}\right) \phi_{n}^{*}\left(S_{t}\right)\right) / B_{0}=$ $c_{i 0}, i=1,2, \ldots, n$.

- Assume $n$ is even. Let $m=n / 2$.

- Then the upper bound for options with strike prices between $\left(K_{2 i-2}\right.$, $\left.K_{2 i-1}\right), i=1,2, \ldots$, is given by a pricing kernel $\varphi_{n}^{* *}\left(S_{t}\right)=a_{0} \frac{\delta\left(S_{t}\right)}{p\left(S_{t}\right)}+$ $b+f_{n}^{* *}\left(S_{t}\right)$, where $p\left(S_{t}\right)$ is the true probability density function, $\delta\left(S_{t}\right)$ is the Dirac function, and $f_{n}^{* *}(x)=$

$$
\begin{array}{cc}
\delta_{m}^{* *}\left(s_{m+1}^{* *}-s_{m}^{* *}\right)+\ldots+\delta_{2}^{* *}\left(s_{3}^{* *}-s_{2}^{* *}\right)+\delta_{1}^{* *}\left(s_{2}^{* *}-x\right), & x<s_{2}^{* *} \\
\ldots \ldots & \\
\delta_{m}^{* *}\left(s_{m+1}^{* *}-x\right), & s_{m}^{* *}<x<s_{m+1}^{* *} \\
0, & x \geq s_{m+1}^{* *},
\end{array}
$$

where $a_{0}, b, s_{2}^{* *}, \ldots, s_{m+1}^{* *}, \delta_{1}^{* *}, \ldots$, and $\delta_{m}^{* *}$ are to be decided such that 


$$
\begin{aligned}
& E\left(\phi_{n}^{* *}\left(S_{t}\right)\right)=1, E\left(S_{t} \phi_{n}^{* *}\left(S_{t}\right)\right) / B_{0}=S_{0} \text {, and } E\left(c_{i}\left(S_{t}\right) \phi_{n}^{* *}\left(S_{t}\right)\right) / B_{0}= \\
& c_{i 0}, i=1,2, \ldots, n . \\
& - \text { The lower bound for options with strike prices between }\left(K_{2 i-2}, K_{2 i-1}\right), \\
& i=1,2, \ldots, \text { is given by a pricing kernel } \varphi_{n}^{*}(x)= \\
& \delta_{m+1}^{*}\left(s_{m+1}^{*}-s_{m}^{*}\right)+\ldots+\delta_{2}^{*}\left(s_{2}^{*}-s_{1}^{*}\right)+\delta_{1}^{*}\left(s_{1}^{*}-x\right), \\
& \ldots \ldots<s_{1}^{*} \\
& \delta_{m+1}^{*}\left(s_{m+1}^{*}-x\right), \\
& 0, \\
& x \in\left(s_{m-1}^{*}, s_{m}^{*}\right) \\
& x \geq s_{m+1}^{*},
\end{aligned}
$$

where $s_{1}^{*}, \ldots, s_{m+1}^{*}, \delta_{1}^{*}, \ldots$, and $\delta_{m+1}^{*}$ are to be decided such that $E\left(\phi_{n}^{*}\left(S_{t}\right)\right)=1, E\left(S_{t} \phi_{n}^{*}\left(S_{t}\right)\right) / B_{0}=S_{0}$, and $E\left(c_{i}\left(S_{t}\right) \phi_{n}^{*}\left(S_{t}\right)\right) / B_{0}=$ $c_{i 0}, i=1,2, \ldots, n$.

Proof: Let $\delta \rightarrow+\infty$; we immediately obtain the result from Lemma 9.

\section{NSD Option Bounds}

In this section we apply the $N$ th order stochastic dominance rule to derive option bounds from concurrently expiring options. According to Ritchken and Kuo (1989), applying $N$ th order stochastic dominance we have such a pricing kernel that its derivatives alternate to be negative and positive up to the $(N-1)$ th order. 


\subsection{Option Bound Problem and Its Dual}

Given the prices of a unit bond, a stock and some European options written on the stock with the same maturity, we want to know the bounds on the price of another option which has the same maturity when $N$ th $(N \geq 3)$ order stochastic dominance rule applies. That is,

$$
\max (\text { or } \min ) E\left(c_{X}\left(S_{T}\right) \phi\left(S_{T}\right)\right) B_{0}
$$

subject to

$$
\begin{gathered}
\phi^{(i)}(x) \geq 0, \text { for even } i \leq N ; \phi^{(i)}(x) \leq 0, \text { for odd } i \leq N \\
E\left(\phi\left(S_{T}\right)=1\right. \\
E\left(S_{T} \phi\left(S_{T}\right)\right) B_{0}=S_{0} \\
E\left(c_{i}\left(S_{T}\right) \phi\left(S_{T}\right)\right) B_{0}=c_{i 0}, \quad i=1, \ldots, n .
\end{gathered}
$$

The dual problem ${ }^{5}$

$$
\min (\text { or } \max ) \alpha_{1} B_{0}+\alpha_{2} S_{0}+\sum_{i=1}^{n} \alpha_{n+2} c_{i 0}
$$

subject to

$$
\begin{aligned}
& \frac{\int_{0}^{S_{T}} \int_{0}^{x_{k}} \ldots \int_{0}^{x_{1}} E\left(\alpha_{1}+\alpha_{2} x+\sum_{i=1}^{n} \alpha_{n+2} c_{i}(x) \mid x<x_{0}\right) \operatorname{Pr}\left(x<x_{0}\right) d x_{0} \ldots d x_{k}}{\int_{0}^{S_{T}} \int_{0}^{x_{k}} \cdots \int_{0}^{x_{1}} \operatorname{Pr}\left(x<x_{0}\right) d x_{0} \ldots d x_{k}} \\
& \geq(\leq) \frac{\int_{0}^{S_{T}} \int_{0}^{x_{k}} \ldots \int_{0}^{x_{1}} E\left(c_{X}(x) \mid x<x_{0}\right) \operatorname{Pr}\left(x<x_{0}\right) d x_{0} \ldots d x_{k}}{\int_{0}^{S_{T}} \int_{0}^{x_{k}} \ldots \int_{0}^{x_{1}} \operatorname{Pr}\left(x<x_{0}\right) d x_{0} \ldots d x_{k}}, \quad \text { for all } S_{T},
\end{aligned}
$$

where $k=N-3$.

\footnotetext{
${ }^{5}$ The proof of the duality is similar to the case of TSD. For brevity, it is omitted.
} 
The above dual problem suggests an $N$ th $(N>3)$ order arbitrage opportunity if the option bound is violated. An $N$ th order arbitrage opportunity can be understood similar to the third order arbitrage opportunity. Obviously, an $N$ th order arbitrage opportunity is weaker than an $(N-1)$ th order arbitrage opportunity, because the weighted average conditional expectations in the case of $(N-1)$ th order are taken weighted average again with the same weights. This means in the case of $N$ th order the conditional expected returns in the states of lower stock prices carry even less weights than in the case of $(N-1)$ th order.

As in the case of TSD, to solve the above two problems we first solve a similar but more general problem in which we assume that not only the $N$ th order stochastic dominance rule applies but also the absolute value of the pricing kernel's $N$ th derivative is bounded above.

We will show in this paper that under this condition, the option bounds are given by pricing kernels which have piecewise constant $(N-2)$ th derivative, where the number of segments of the $(N-2)$ th derivative depends on the number of observed options. Moreover, we will see that for an even number of observed options the pricing kernel which gives the option bounds has a certain pattern while for an odd number of observed options the pricing kernel which gives option bounds has a different pattern. Thus in order to explain the solutions more clearly we start with the case where we have only one observed option price then continue with the case where we have two observed options. In the end we solve the general case where we have $n$ observed options. 


\subsection{The Case with One Observed Option}

In this subsection we deal with the case where we observe the price of one concurrently expiring option.

We first derive the option bounds under the assumption that the third order stochastic dominance rule applies and the absolute value of the pricing kernel's $(N-2)$ th derivative is bounded from above.

Lemma 8 Assume the pricing kernel $\phi(x)$ satisfies the Nth order stochastic dominance rule, i.e., $\phi^{(i)}(x) \geq 0$, for even $i<N, \phi^{(i)}(x) \leq 0$, for odd $i<N$, and $\left|\phi^{(N-1)}(x)\right|$ is increasing in $x$. Assume $\left|\phi^{(N-2)}(x)\right|$ is bounded above by $\delta$. Assume the current price of a unit bond is $B_{0}$, the current price of the underlying stock is $S_{0}$ and the current price of an option with strike price $K$ is $c_{K 0}$.

- Then the upper option bound is given by a pricing kernel $\left(\phi_{1}^{* *}(x)\right)$ that has a three-segmented and piecewise constant $(N-2)$ th derivative, where its $(N-2)$ th derivative is equal to $-\delta$ for $x<s_{1}^{* *},-\delta^{* *}$ for $x \in\left(s_{1}^{* *}, s_{2}^{* *}\right)$, and zero for $x>s_{2}^{* *}$, and its value is zero at its right tail. More precisely

$$
\phi_{1}^{* *}(x)=\left\{\begin{array}{cc}
\delta \frac{\left(s_{1}^{* *}-x\right)^{N-2}}{(N-2) !}+\delta^{* *} \sum_{1}^{N-2} \frac{\left(s_{2}^{* *}-s_{1}^{* *}\right)^{i}}{i !} \frac{\left(s_{1}^{* *}-x\right)^{(N-2-i)}}{(N-2-i) !}, & x<s_{1}^{* *} \\
\frac{\delta^{* *}}{(N-2) !}\left(s_{2}^{* *}-x\right)^{N-2}, & x \in\left(s_{1}^{* *}, s_{2}^{* *}\right) \\
0, & x \geq s_{2}^{* *},
\end{array}\right.
$$

where $s_{1}^{* *}, s^{* *}$, and $\delta^{* *}$ are to be decided such that $E\left(\phi_{1}^{* *}\left(S_{t}\right)\right)=1$, $E\left(S_{t} \phi_{1}^{* *}\left(S_{t}\right)\right) / B_{0}=S_{0}$, and $E\left(c_{K}\left(S_{t}\right) \phi_{1}^{* *}\left(S_{t}\right)\right) / B_{0}=c_{K 0}$.

- The lower option bound is given by a pricing kernel $\left(\phi_{0}^{*}(x)\right)$ that has a 
two-segmented and piecewise constant ( $N$-2)th derivative. More precisely its (N-2)th derivative is equal to $-\delta^{*}$ for $x<s^{*}$, and zero for $x>s^{*}$.

That is,

$$
\phi_{1}^{*}(x)=\left\{\begin{array}{cc}
b+\frac{\delta^{*}}{(N-2) !}\left(s^{*}-x\right)^{N-2}, & x<s^{*} \\
b, & x \geq s^{*},
\end{array}\right.
$$

where $b, \delta^{*}$, and $s^{*}$ are to be decided such that $E\left(\phi_{1}^{*}\left(S_{t}\right)\right)=1, E\left(S_{t} \phi_{0}^{*}\right.$ $\left.\left(S_{t}\right)\right) / B_{0}=S_{0}$, and $E\left(c_{K}\left(S_{t}\right) \phi_{1}^{*}\left(S_{t}\right)\right) / B_{0}=c_{K 0}$.

Proof: From Lemma 2 we need only prove that the true pricing kernel intersects the pricing kernels that give the option bounds exactly three times and then examine which one has a fatter left tail.

We first examine $\phi_{1}^{* *}$. Note it has a three-segmented and piecewise constant $(N-2)$ th derivative. More precisely its $(N-2)$ th derivative is equal to $-\delta$ for $x<s_{1}^{* *},-\delta^{* *}$ for $x \in\left(s_{1}^{* *}, s_{2}^{* *}\right)$, and zero for $x>s_{2}^{* *}$, and its value is zero at its right tail. Obviously we must have $0<\delta^{* *}<\delta$. Otherwise the true pricing kernel will intersect $\phi_{1}^{* *}$ at most twice. In this case, applying Lemma 1, we find that the two pricing kernels cannot give the same observed option price. From this, we can immediately conclude that the true pricing kernel intersects $\phi_{1}^{* *}$ exactly three times. It is not difficult to verify that $\phi_{1}^{* *}$ has fatter left tail. For $\phi_{1}^{*}$ the proof is similar. Q.E.D.

Proposition 4 Assume the pricing kernel $\phi(x)$ satisfies the Nth order stochastic dominance rule, i.e., $\phi^{(i)}(x) \geq 0$, for even $i<N, \phi^{(i)}(x) \leq 0$, for odd $i<N$, and $\left|\phi^{(N-1)}(x)\right|$ is increasing in $x$. Assume the current price of a unit bond is $B_{0}$, the current price of the underlying stock is $S_{0}$ and the current price of an 
option with strike price $K$ is $c_{K 0}$.

- Then the upper option bound is given by the pricing kernel $\varphi_{1}^{* *}\left(S_{t}\right)=$ $a_{0} \frac{\delta\left(S_{t}\right)}{p\left(S_{t}\right)}+f_{1}^{* *}\left(S_{t}\right)$, where $p\left(S_{t}\right)$ is the true probability density function and $\delta\left(S_{t}\right)$ is the Dirac function and

$$
f_{1}^{* *}\left(S_{t}\right)=\left\{\begin{array}{cc}
\frac{\delta^{* *}}{(N-2) !}\left(s^{* *}-x\right)^{N-2}, & x<s^{* *} \\
0, & x \geq s^{* *},
\end{array}\right.
$$

where $a_{0}, s^{* *}$, and $\delta^{* *}$ are to be decided such that $E\left(\varphi_{1}^{* *}\left(S_{t}\right)\right)=1, E\left(S_{t} \varphi_{1}^{* *}\right.$ $\left.\left(S_{t}\right)\right) / B_{0}=S_{0}$, and $E\left(c_{K}\left(S_{t}\right) \varphi_{1}^{* *}\left(S_{t}\right)\right) / B_{0}=c_{K 0}$.

- The lower option bound is given by the pricing kernel $\varphi_{1}^{*}(x)=\phi_{1}^{*}(x)$.

\subsection{The Case with Two Observed Options}

In this subsection we deal with the case where we observe the prices of two concurrently expiring options.

We first derive the option bounds under the assumption that the third order stochastic dominance rule applies and the absolute value of the pricing kernel's $(N-2)$ th derivative is bounded from above.

Lemma 9 Assume the pricing kernel $\phi(x)$ satisfies the Nth order stochastic dominance rule, i.e., $\phi^{(i)}(x) \geq 0$, for even $i<N$, $\phi^{(i)}(x) \leq 0$, for odd $i<N$, and $\left|\phi^{(N-1)}(x)\right|$ is increasing in $x$. Assume $\left|\phi^{(N-2)}(x)\right|$ is bounded above by $\delta$. Assume the current price of a unit bond is $B_{0}$, the current price of the underlying stock is $S_{0}$, and the current prices of two options with strike prices $K_{1}$ and $K_{2}$ are $c_{10}$ and $c_{20}$ respectively. 
- Then the upper bound for an option with a strike price below $K_{1}$ or above $K_{2}$ is given by a pricing kernel $\left(\phi_{2}^{* *}(x)\right)$ that has a three-segmented and piecewise constant ( $N$-2)th derivative, where its ( $N$-2)th derivative is equal to $-\delta$ for $x<s_{1}^{* *},-\delta^{* *}$ for $x \in\left(s_{1}^{* *}, s_{2}^{* *}\right)$, and zero for $x>s_{2}^{* *}$, and its value is a positive constant $b$ at its right tail. More precisely, $\phi_{2}^{* *}(x)=b+$

$$
\left\{\begin{array}{cc}
\frac{\delta}{(N-2) !}\left(s_{1}^{* *}-x\right)^{N-2}+\delta^{* *} \sum_{1}^{N-2} \frac{\left(s_{2}^{* *}-s_{1}^{* *}\right)^{i}}{i !} \frac{\left(s_{1}^{* *}-x\right)^{(N-2-i)}}{(N-2-i) !}, & x<s_{1}^{* *} \\
\frac{\delta^{* *}}{(N-2) !}\left(s_{2}^{* *}-x\right)^{N-2}, & s_{1}^{* *}<x<s_{2}^{* *} \\
0, & x \geq s_{2}^{* *},
\end{array}\right.
$$

where $b, s_{1}^{* *}, s_{2}^{* *}$, and $\delta^{* *}$ are to be decided such that $E\left(\phi_{1}^{* *}\left(S_{t}\right)\right)=1$, $E\left(S_{t} \phi_{1}^{* *}\left(S_{t}\right)\right) / B_{0}=S_{0}$, and $E\left(c_{i}\left(S_{t}\right) \phi_{1}^{* *}\left(S_{t}\right)\right) / B_{0}=c_{i 0}, i=1,2$.

- The lower bound for an option with a strike price below $K_{1}$ or above $K_{2}$ is given by a pricing kernel $\left(\phi_{2}^{*}(x)\right)$ that has a three-segmented and piecewise constant (N-2)th derivative. More precisely its (N-2)th derivative is equal to $-\delta_{1}^{*}$ for $x<s_{1}^{*},-\delta_{2}^{*}$ for $x \in\left(s_{1}^{*}, s_{2}^{*}\right)$, and zero for $x>s_{2}^{*}$. That is,

$$
\phi_{2}^{*}(x)=\left\{\begin{array}{cc}
\frac{\delta_{1}^{*}}{(N-2) !}\left(s_{1}^{*}-x\right)^{N-2}+\delta_{2}^{*} \sum_{1}^{N-2} \frac{\left(s_{2}^{*}-s_{1}^{*}\right)^{i}}{i !} \frac{\left(s_{1}^{*}-x\right)^{(N-2-i)}}{(N-2-i) !}, & x<s_{1}^{*} \\
\frac{\delta_{2}^{*}}{(N-2) !}\left(s_{2}^{*}-x\right)^{N-2}, & x \in\left(s_{1}^{*}, s_{2}^{*}\right) \\
0, & x \geq s_{2}^{*},
\end{array}\right.
$$

where $s_{1}^{*}, s_{2}^{*}, \delta_{1}^{*}$, and $\delta_{2}^{*}$ are to be decided such that $E\left(\phi_{1}^{*}\left(S_{t}\right)\right)=1$, $E\left(S_{t} \phi_{1}^{*}\left(S_{t}\right)\right) / B_{0}=S_{0}$, and $E\left(c_{i}\left(S_{t}\right) \phi_{1}^{*}\left(S_{t}\right)\right) / B_{0}=c_{i 0}, i=1,2$.

Proof: From Lemma 4 we need only prove that the true pricing kernel intersects the pricing kernels that give the option bounds exactly four times and then examine which one has a fatter left tail. 
We first examine $\phi_{2}^{* *}$. Note it has a three-segmented and piecewise constant $(N-2)$ th derivative. More precisely its $(N-2)$ th derivative is equal to $-\delta$ for $x<s_{1}^{* *},-\delta^{* *}$ for $x \in\left(s_{1}^{* *}, s_{2}^{* *}\right)$, and zero for $x>s_{2}^{* *}$, and its value is a positive constant $b$ at its right tail.

Obviously we must have $0<\delta^{* *}<\delta$ and $b>\inf _{x} \phi(x)$, where $\phi(x)$ is the true pricing kernel. Otherwise the true pricing kernel will intersect $\phi_{2}^{* *}$ at most three times. In this case, applying Lemma 2, we find that the two pricing kernels cannot give the same two observed option prices. From this, we can immediately conclude that the true pricing kernel intersects $\phi_{2}^{* *}$ exactly four times. It is not difficult to verify that $\phi_{2}^{* *}$ has fatter left tail. For $\phi_{2}^{*}$ the proof is similar. Q.E.D.

Proposition 5 Assume the pricing kernel $\phi(x)$ satisfies the Nth order stochastic dominance rule, i.e., $\phi^{(i)}(x) \geq 0$, for even $i<N, \phi^{(i)}(x) \leq 0$, for odd $i<N$, and $\left|\phi^{(N-1)}(x)\right|$ is increasing in $x$. Assume the current price of a unit bond is $B_{0}$, the current price of the underlying stock is $S_{0}$, and the current prices of two options with strike prices $K_{1}$ and $K_{2}$ are $c_{10}$ and $c_{20}$ respectively.

- Then the upper bound for an option with a strike price below $K_{1}$ or above $K_{2}$ is given by the pricing kernel $\varphi_{2}^{* *}\left(S_{t}\right)=a_{0} \frac{\delta\left(S_{t}\right)}{p\left(S_{t}\right)}+f_{2}^{* *}\left(S_{t}\right)$, where $p\left(S_{t}\right)$ is the true probability density function and $\delta\left(S_{t}\right)$ is the Dirac function and

$$
f_{2}^{* *}\left(S_{t}\right)=\left\{\begin{array}{cc}
b+\frac{\delta^{* *}}{(N-2) !}\left(s^{* *}-x\right)^{N-2}, & x<s^{* *} \\
b, & x \geq s^{* *},
\end{array}\right.
$$

where $a_{0}, b, s^{* *}$, and $\delta^{* *}$ are to be decided such that $E\left(\varphi_{2}^{* *}\left(S_{t}\right)\right)=1$, $E\left(S_{t} \varphi_{2}^{* *}\left(S_{t}\right)\right) / B_{0}=S_{0}$, and $E\left(c_{i}\left(S_{t}\right) \varphi_{2}^{* *}\left(S_{t}\right)\right) / B_{0}=c_{i 0}, i=1,2$. 
- The lower bound for an option with a strike price below $K_{1}$ or above $K_{2}$ is given by the pricing kernel $\varphi_{2}^{*}(x)=\phi_{2}^{*}(x)$.

\subsection{The General Case}

In this subsection we deal with the case where we observe the prices of $n$ concurrently expiring options.

We first derive the option bounds under the assumption that the third order stochastic dominance rule applies and the absolute value of the pricing kernel's $(N-2)$ th derivative is bounded from above.

Lemma 10 Assume the pricing kernel $\phi(x)$ satisfies the Nth order stochastic dominance rule, i.e., $\phi^{(i)}(x) \geq 0$, for even $i<N$, $\phi^{(i)}(x) \leq 0$, for odd $i<N$, and $\left|\phi^{(N-1)}(x)\right|$ is increasing in $x$. Assume $\left|\phi^{(N-2)}(x)\right|$ is bounded above by $\delta$. Assume the current price of a unit bond is $B_{0}$, the current price of the underlying stock is $S_{0}$, and the current prices of $n$ options with strike prices $K_{1}, \ldots$, and $K_{n}$ are $c_{10}, \ldots$, and $c_{n 0}$ respectively. Let $K_{0}=0$ and $K_{n+1}=+\infty$.

- Assume $n$ is odd. Let $m=(n+1) / 2$.

- Then the upper bound for options with strike prices between $\left(K_{2 i-2}\right.$, $\left.K_{2 i-1}\right), i=1,2, \ldots$, is given by a pricing kernel $\left(\phi_{n}^{* *}(x)\right)$ that has a $(m+2)$-segmented and piecewise constant (N-2)th derivative, where the absolute of value of its (N-2)th derivative is equal to $\delta$ for $x<$ $s_{1}^{* *}, \delta_{1}^{* *}$ for $x \in\left(s_{1}^{* *}, s_{2}^{* *}\right), \ldots, \delta_{m}^{* *}$ for $x \in\left(s_{m}^{* *}, s_{m+1}^{* *}\right)$, and zero for $x>s_{m+1}^{* *}$, and its value at its right tail is 0. More precisely, 
$\phi_{n}^{* *}(x)=\phi_{n i}^{* *}(x)$, for $x \in\left(s_{i-1}^{* *}, s_{i}^{* *}\right), i=1, \ldots, m+2$, where $s_{0}^{* *}=0$, $s_{m+2}^{* *}=+\infty, \phi_{n(m+2)}^{* *}(x)=0, \phi_{n(m+1)}^{* *}(x)=\frac{\delta_{m}^{* *}}{(N-2) !}\left(s_{m+1}^{* *}-x\right)^{N-2}$, and for $i=1, \ldots m$,

$$
\begin{aligned}
\phi_{n i}^{* *}(x)= & \phi_{n(i+2)}^{* *}\left(s_{i+1}^{* *}\right)+\delta_{i-1}^{* *} \frac{\left(s_{i}^{* *}-x\right)^{N-2}}{(N-2) !} \\
& +\delta_{i}^{* *} \sum_{1}^{N-2} \frac{\left(s_{i+1}^{* *}-s_{i}^{* *}\right)^{j}}{j !} \frac{\left(s_{i}^{* *}-x\right)^{(N-2-j)}}{(N-2-j) !},
\end{aligned}
$$

where $\delta_{0}^{* *}=\delta$ while $s_{1}^{* *}, \ldots, s_{m+1}^{* *}, \delta_{1}^{* *}, \ldots$, and $\delta_{m}^{* *}$ are to be decided such that $E\left(\phi_{n}^{* *}\left(S_{t}\right)\right)=1, E\left(S_{t} \phi_{n}^{* *}\left(S_{t}\right)\right) / B_{0}=S_{0}$, and $E\left(c_{i}\left(S_{t}\right) \phi_{n}^{* *}\right.$ $\left.\left(S_{t}\right)\right) / B_{0}=c_{i 0}, i=1,2, \ldots, n$.

- The lower bound for options with strike prices between $\left(K_{2 i-2}, K_{2 i-1}\right)$, $i=1,2, \ldots$, is given by a pricing kernel $\left(\phi_{\text {on }}^{*}(x)\right)$ that has a $(m+1)$ segmented and piecewise constant ( $N$-2)th derivative, where the absolute of value of its (N-2)th derivative is equal to $\delta_{1}^{*}$ for $x<s_{1}^{*}$, $\ldots, \delta_{m}^{*}$ for $x \in\left(s_{m-1}^{*}, s_{m}^{*}\right)$, and zero for $x>s_{m}^{*}$. More precisely, $\phi_{o n}^{*}(x)=\phi_{n i}^{*}(x)$, for $x \in\left(s_{i-1}^{*}, s_{i}^{*}\right), i=1, \ldots, m+1$, where $s_{0}^{*}=0$, $s_{m+1}^{*}=+\infty, \phi_{n(m+1)}^{*}(x)=b, \phi_{n m}^{*}(x)=b+\frac{\delta_{m}^{*}}{(N-2) !}\left(s_{m}^{*}-x\right)^{N-2}$, and for $i=1, \ldots, m-1$,

$$
\begin{aligned}
\phi_{n i}^{*}(x)= & \phi_{n(i+2)}^{*}\left(s_{i+1}^{*}\right)+\delta_{i}^{*} \frac{\left(s_{i}^{*}-x\right)^{N-2}}{(N-2) !} \\
& +\delta_{i+1}^{*} \sum_{1}^{N-2} \frac{\left(s_{i+1}^{*}-s_{i}^{*}\right)^{j}}{j !} \frac{\left(s_{i}^{*}-x\right)^{(N-2-j)}}{(N-2-j) !},
\end{aligned}
$$

where $b, s_{1}^{*}, \ldots, s_{m}^{*}, \delta_{1}^{*}, \ldots$, and $\delta_{m}^{*}$ are to be decided such that $E\left(\phi_{o n}^{*}\left(S_{t}\right)\right)=1, E\left(S_{t} \phi_{o n}^{*}\left(S_{t}\right)\right) / B_{0}=S_{0}$, and $E\left(c_{i}\left(S_{t}\right) \phi_{o n}^{*}\left(S_{t}\right)\right) / B_{0}=$ $c_{i 0}, i=1,2, \ldots, n$. 
- Assume $n$ is even. Let $m=n / 2$.

- Then the upper bound for options with strike prices between $\left(K_{2 i-2}\right.$, $\left.K_{2 i-1}\right), i=1,2, \ldots$, is given by a pricing kernel $\left(\phi_{n}^{* *}(x)\right)$ that has a $(m+2)$-segmented and piecewise constant (N-2)th derivative, where the absolute value of its (N-2)th derivative is equal to $\delta$ for $x<$ $s_{1}^{* *}, \delta_{1}^{* *}$ for $x \in\left(s_{1}^{* *}, s_{2}^{* *}\right), \ldots, \delta_{m}^{* *}$ for $x \in\left(s_{m}^{* *}, s_{m+1}^{* *}\right)$, and zero for $x>s_{m+1}^{* *}$. More precisely, $\phi_{n}^{* *}(x)=\phi_{n i}^{* *}(x)$, for $x \in\left(s_{i-1}^{* *}, s_{i}^{* *}\right)$, $i=1, \ldots, m+2$, where $s_{0}^{* *}=0, s_{m+2}^{* *}=+\infty, \phi_{n(m+2)}^{* *}(x)=b$, $\phi_{n(m+1)}^{* *}(x)=b+\frac{\delta_{m}^{* *}}{(N-2) !}\left(s_{m+1}^{* *}-x\right)^{N-2}$, and for $i=1, \ldots m$,

$$
\begin{aligned}
\phi_{n i}^{* *}(x)= & \phi_{n(i+2)}^{* *}\left(s_{i+1}^{* *}\right)+\delta_{i-1}^{* *} \frac{\left(s_{i}^{* *}-x\right)^{N-2}}{(N-2) !} \\
& +\delta_{i}^{* *} \sum_{1}^{N-2} \frac{\left(s_{i+1}^{* *}-s_{i}^{* *}\right)^{j}}{j !} \frac{\left(s_{i}^{* *}-x\right)^{(N-2-j)}}{(N-2-j) !},
\end{aligned}
$$

where $\delta_{0}^{* *}=\delta$ while $b, s_{1}^{* *}, \ldots, s_{m+1}^{* *}, \delta_{1}^{* *}, \ldots$, and $\delta_{m}^{* *}$ are to be decided such that $E\left(\phi_{n}^{* *}\left(S_{t}\right)\right)=1, E\left(S_{t} \phi_{n}^{* *}\left(S_{t}\right)\right) / B_{0}=S_{0}$, and $E\left(c_{i}\left(S_{t}\right) \phi_{n}^{* *}\right.$ $\left.\left(S_{t}\right)\right) / B_{0}=c_{i 0}, i=1,2, \ldots, n$.

- The lower bound for options with strike prices between $\left(K_{2 i-2}, K_{2 i-1}\right)$, $i=1,2, \ldots$, is given by a pricing kernel $\left(\phi_{e n}^{*}(x)\right)$ that has a $(m+2)$ segmented and piecewise constant (N-2)th derivative, where the absolute of value of its ( $N$-2)th derivative is equal to $\delta_{1}^{*}$ for $x<s_{1}^{*}, \ldots$, $\delta_{m+1}^{*}$ for $x \in\left(s_{m}^{*}, s_{m+1}^{*}\right)$, and zero for $x>s_{m+1}^{*}$. More precisely, $\phi_{e n}^{*}(x)=\phi_{n i}^{*}(x)$, for $x \in\left(s_{i-1}^{*}, s_{i}^{*}\right), i=1, \ldots, m+2$, where $s_{0}^{*}=0$, $s_{m+2}^{*}=+\infty, \phi_{n(m+2)}^{*}(x)=0, \phi_{n(m+1)}^{*}(x)=\frac{\delta_{m+1}^{*}}{(N-2) !}\left(s_{m+1}^{*}-x\right)^{N-2}$, 
and for $i=1, \ldots, m$,

$$
\begin{aligned}
\phi_{n i}^{*}(x)= & \phi_{n(i+2)}^{*}\left(s_{i+1}^{*}\right)+\delta_{i}^{*} \frac{\left(s_{i}^{*}-x\right)^{N-2}}{(N-2) !} \\
& +\delta_{i+1}^{*} \sum_{1}^{N-2} \frac{\left(s_{i+1}^{*}-s_{i}^{*}\right)^{j}}{j !} \frac{\left(s_{i}^{*}-x\right)^{(N-2-j)}}{(N-2-j) !},
\end{aligned}
$$

where $s_{1}^{*}, \ldots, s_{m+1}^{*}, \delta_{1}^{*}, \ldots$, and $\delta_{m+1}^{*}$ are to be decided such that $E\left(\phi_{e n}^{*}\left(S_{t}\right)\right)=1, E\left(S_{t} \phi_{e n}^{*}\left(S_{t}\right)\right) / B_{0}=S_{0}$, and $E\left(c_{i}\left(S_{t}\right) \phi_{e n}^{*}\left(S_{t}\right)\right) / B_{0}=$ $c_{i 0}, i=1,2, \ldots, n$.

Proof: From Lemma 6 we need only prove that the true pricing kernel intersects the pricing kernels that give the option bounds exactly $(n+2)$ times and then examine which one has a fatter left tail.

We first examine $\phi_{n}^{* *}$. Assume $n$ is odd. Note it has a $(\mathrm{m}+2)$-segmented and piecewise constant $(N-2)$ th derivative, where $m=(n+1) / 2$. More precisely its $(N-2)$ th derivative is equal to $-\delta$ for $x<s_{1}^{* *},-\delta_{1}^{* *}$ for $x \in\left(s_{1}^{* *}, s_{2}^{* *}\right), \ldots$, $-\delta_{m}^{* *}$ for $x \in\left(s_{m}^{* *}, s_{m+1}^{* *}\right)$, and zero for $x>s_{m+1}^{* *}$, and its value at its right tail is 0 .

Obviously we must have $\delta>\delta_{1}^{* *}>\ldots \delta_{m}^{* *}>0$. Otherwise the true pricing kernel will intersect $\phi_{n}^{* *}$ at most $n+1$ times. In this case, applying Lemma 6 , we find that the two pricing kernels cannot give the same $n$ observed option prices. From this, we can immediately conclude that the true pricing kernel intersects $\phi_{n}^{* *}$ exactly $n+2$ times. It is not difficult to verify that $\phi_{n}^{* *}$ has fatter left tail.

Assume $n$ is even. Note it has a $(\mathrm{m}+2)$-segmented and piecewise constant $(N-2)$ th derivative, where $m=n / 2$. More precisely, the absolute value of its $(N-2)$ th derivative is equal to $\delta$ for $x<s_{1}^{* *}, \delta_{1}^{* *}$ for $x \in\left(s_{1}^{* *}, s_{2}^{* *}\right), \ldots, \delta_{m}^{* *}$ 
for $x \in\left(s_{m}^{* *}, s_{m+1}^{* *}\right)$, and zero for $x>s_{m+1}^{* *}$, and its value at its right tail is a positive constant $b$.

Obviously we must have $\delta>\delta_{1}^{* *}>\ldots \delta_{m}^{* *}>0$ and $b>\inf _{x} \phi(x)$, where $\phi(x)$ is the true pricing kernel. Otherwise the true pricing kernel will intersect $\phi_{n}^{* *}$ at most $n+1$ times. In this case, applying Lemma 6 , we find that the two pricing kernels cannot give the same $n$ observed option prices. From this, we can immediately conclude that the true pricing kernel intersects $\phi_{n}^{* *}$ exactly $n+2$ times. It is not difficult to verify that $\phi_{n}^{* *}$ has fatter left tail.

For $\phi_{n}^{*}$ the proof is similar. Q.E.D.

Proposition 6 Assume the pricing kernel $\phi(x)$ satisfies the Nth order stochastic dominance rule, i.e., $\phi^{(i)}(x) \geq 0$, for even $i<N, \phi^{(i)}(x) \leq 0$, for odd $i<N$, and $\left|\phi^{(N-1)}(x)\right|$ is increasing in $x$. Assume $\left|\phi^{(N-2)}(x)\right|$ is bounded above by $\delta$. Assume the current price of a unit bond is $B_{0}$, the current price of the underlying stock is $S_{0}$, and the current prices of $n$ options with strike prices $K_{1}, \ldots$, and $K_{n}$ are $c_{10}, \ldots$, and $c_{n 0}$ respectively. Let $K_{0}=0$ and $K_{n+1}=+\infty$.

1. Assume $n$ is odd. Let $m=(n+1) / 2$.

(a) Then the upper bound for options with strike prices between $\left(K_{2 i-2}\right.$, $\left.K_{2 i-1}\right), i=1,2, \ldots$, is given by the pricing kernel $\varphi_{n}^{* *}\left(S_{t}\right)=a_{0} \frac{\delta\left(S_{t}\right)}{p\left(S_{t}\right)}+$ $f_{n}^{* *}\left(S_{t}\right)$, where $p\left(S_{t}\right)$ is the true probability density function, $\delta\left(S_{t}\right)$ is the Dirac function, and $f_{n}^{* *}(x)$ has a $(m+1)$-segmented and piecewise constant (N-2)th derivative, where the absolute of value of its (N-2)th derivative is equal to $\delta_{1}^{* *}$ for $x<s_{1}^{* *}, \ldots, \delta_{m}^{* *}$ for $x \in\left(s_{m-1}^{* *}, s_{m}^{* *}\right)$, and 
zero for $x>s_{m}^{* *}$. More precisely, $f_{n}^{* *}(x)=f_{n i}^{* *}(x)$, for $x \in\left(s_{i-1}^{* *}, s_{i}^{* *}\right)$, $i=1, \ldots, m+1$, where $s_{0}^{* *}=0, s_{m+1}^{* *}=+\infty, f_{n(m+1)}^{* *}(x)=0$, $f_{n m}^{* *}(x)=\frac{\delta_{m}^{* *}}{(N-2) !}\left(s_{m}^{* *}-x\right)^{N-2}$, and for $i=1, \ldots, m-1$,

$$
\begin{aligned}
f_{n i}^{* *}(x)= & f_{n(i+2)}^{* *}\left(s_{i+1}^{* *}\right)+\delta_{i}^{* *} \frac{\left(s_{i}^{* *}-x\right)^{N-2}}{(N-2) !} \\
& +\delta_{i+1}^{* *} \sum_{1}^{N-2} \frac{\left(s_{i+1}^{* *}-s_{i}^{* *}\right)^{j}}{j !} \frac{\left(s_{i}^{* *}-x\right)^{(N-2-j)}}{(N-2-j) !}
\end{aligned}
$$

where $a_{0}, s_{1}^{* *}, \ldots, s_{m}^{* *}, \delta_{1}^{* *}, \ldots$, and $\delta_{m}^{* *}$ are to be decided such that $E\left(\phi_{n}^{* *}\left(S_{t}\right)\right)=1, E\left(S_{t} \phi_{n}^{* *}\left(S_{t}\right)\right) / B_{0}=S_{0}$, and $E\left(c_{i}\left(S_{t}\right) \phi_{n}^{* *}\left(S_{t}\right)\right) / B_{0}=$ $c_{i 0}, i=1,2, \ldots, n$.

(b) The lower bound for options with strike prices between $\left(K_{2 i-2}, K_{2 i-1}\right)$, $i=1,2, \ldots$, is given by the pricing kernel $\varphi_{n}^{*}(x)=\phi_{o n}^{*}(x)$, which is derived in the above proposition.

2. Assume $n$ is even. Let $m=n / 2$.

(a) Then the upper bound for options with strike prices between $\left(K_{2 i-2}\right.$, $\left.K_{2 i-1}\right), i=1,2, \ldots$, is given by a pricing kernel $\varphi_{n}^{* *}\left(S_{t}\right)=a_{0} \frac{\delta\left(S_{t}\right)}{p\left(S_{t}\right)}+$ $f_{n}^{* *}\left(S_{t}\right)$, where $p\left(S_{t}\right)$ is the true probability density function, $\delta\left(S_{t}\right)$ is the Dirac function, and $f_{n}^{* *}(x)$ has a $(m+1)$-segmented and piecewise constant (N-2)th derivative, where the absolute of value of its (N-2)th derivative is equal to $\delta_{1}^{* *}$ for $x<s_{1}^{* *}, \ldots, \delta_{m}^{* *}$ for $x \in\left(s_{m-1}^{* *}, s_{m}^{* *}\right)$, and zero for $x>s_{m}^{* *}$. More precisely, $f_{n}^{* *}(x)=f_{n i}^{* *}(x)$, for $x \in\left(s_{i-1}^{* *}, s_{i}^{* *}\right)$, $i=1, \ldots, m+1$, where $s_{0}^{* *}=0, s_{m+1}^{* *}=+\infty, f_{n(m+1)}^{* *}(x)=b$, 


$$
\begin{aligned}
& f_{n m}^{* *}(x)=b+\frac{\delta_{m}^{* *}}{(N-2) !}\left(s_{m}^{* *}-x\right)^{N-2}, \text { and for } i=1, \ldots, m-1, \\
& \qquad \begin{aligned}
f_{n i}^{* *}(x)= & f_{n(i+2)}^{* *}\left(s_{i+1}^{* *}\right)+\delta_{i}^{* *} \frac{\left(s_{i}^{* *}-x\right)^{N-2}}{(N-2) !} \\
& +\delta_{i+1}^{* *} \sum_{1}^{N-2} \frac{\left(s_{i+1}^{* *}-s_{i}^{* *}\right)^{j}}{j !} \frac{\left(s_{i}^{* *}-x\right)^{(N-2-j)}}{(N-2-j) !}
\end{aligned}
\end{aligned}
$$

where $a_{0}, b, s_{1}^{* *}, \ldots, s_{m}^{* *}, \delta_{1}^{* *}, \ldots$, and $\delta_{m}^{* *}$ are to be decided such that $E\left(\phi_{n}^{* *}\left(S_{t}\right)\right)=1, E\left(S_{t} \phi_{n}^{* *}\left(S_{t}\right)\right) / B_{0}=S_{0}$, and $E\left(c_{i}\left(S_{t}\right) \phi_{n}^{* *}\left(S_{t}\right)\right) / B_{0}=$ $c_{i 0}, i=1,2, \ldots, n$.

(b) The lower bound for options with strike prices between $\left(K_{2 i-2}, K_{2 i-1}\right)$, $i=1,2, \ldots$, is given by a pricing kernel $\varphi_{n}^{*}(x)=\phi_{e n}^{*}$, which is derived in the above proposition.

\section{The Arbitrage Portfolios}

Using a method similar to the one use by Huang (2000b) we can derive the arbitrage portfolios when the $N$ th order stochastic dominance option bounds are violated. We first introduce the following notation: Let $k=N-3$. We use $\bar{s}_{j}^{(N)}$ to denote

$$
\frac{\int_{0}^{s_{j}} \int_{0}^{x_{k}} \ldots \int_{0}^{x_{1}} E\left(S_{t} \mid S_{t}<x_{0}\right) \operatorname{Pr}\left(S_{t}<x_{0}\right) d x_{0} \ldots d x_{k}}{\int_{0}^{s_{j}} \int_{0}^{x_{k}} \cdots \int_{0}^{x_{1}} \operatorname{Pr}\left(S_{t}<x_{0}\right) d x_{0} \ldots d x_{k}}
$$

Given a function $f(x)$, we use $\bar{f}_{l_{j}}^{(N)}$ to denote the following

$$
\frac{\int_{0}^{s_{j}} \int_{0}^{x_{k}} \ldots \int_{0}^{x_{1}} E\left(f\left(S_{t}\right) \mid S_{t}<x_{0}\right) \operatorname{Pr}\left(S_{t}<x_{0}\right) d x_{0} \ldots d x_{k}}{\int_{0}^{s_{j}} \int_{0}^{x_{k}} \cdots \int_{0}^{x_{1}} \operatorname{Pr}\left(S_{t}<x_{0}\right) d x_{0} \ldots d x_{k}}
$$

We also use $\left(\bar{c}_{l_{j}}^{(N) X}\right)^{\prime}$ to denote

$$
\left.\frac{d}{d y} \frac{\int_{0}^{y} \int_{0}^{x_{k}} \ldots \int_{0}^{x_{1}} E\left(c_{l_{j}}^{X}\left(S_{t}\right) \mid S_{t}<x_{0}\right) \operatorname{Pr}\left(S_{t}<x_{0}\right) d x_{0} \ldots d x_{k}}{\int_{0}^{y} \int_{0}^{x_{k}} \ldots \int_{0}^{x_{1}} \operatorname{Pr}\left(S_{t}<x_{0}\right) d x_{0} \ldots d x_{k}}\right|_{y=s_{j}} .
$$


We have the following result.

Proposition 7 Assume the pricing kernel is decreasing in $S_{t}$. Assume the price of a unit bond is $B_{0}$, the underlying stock price is $S_{0}$, and the prices of $n$ options with strike prices $K_{1}, K_{2}, \ldots, K_{n}$ are $c_{0}^{1}, c_{0}^{2}, \ldots$, and $c_{0}^{n}$ respectively.

- Assume $n$ is odd. Let $m=(n+1) / 2$.

- For options with strike prices $X$ between $\left(K_{2 i-2}, K_{2 i-1}\right), i=1,2, \ldots$, when its lower bound is violated the arbitrage portfolio is given by

$$
\alpha_{i}=(-1)^{i}\left[\sum_{1}^{m}\left(\left(\bar{c}_{l_{v}}^{(N) X}\right)^{\prime} A_{2 v, i}-\bar{c}_{l_{v}}^{(N) X} A_{2 v-1, i}\right)-\bar{c}_{\Lambda}^{(N) X} A_{n+2, i}\right] /|A|,
$$

$i=1, \ldots, n+2$, where $s_{l_{1}}, \ldots, s_{l_{m}}$, are determined by $1(b)$ in Proposition 6 and $A$ is given by

$$
\left\{\begin{array}{ccccc}
1 & \bar{s}_{l_{1}}^{(N)} & \bar{c}_{l_{1}}^{1} & \ldots & \bar{c}_{l_{1}}^{(N) n} \\
0 & \left(\bar{s}_{l_{1}}^{(N)}\right)^{\prime} & \left(\bar{c}_{l_{1}}^{(N) 1}\right)^{\prime} & \ldots & \left(\bar{c}_{l_{1}}^{(N) n}\right)^{\prime} \\
\vdots & \vdots & \vdots & \vdots & \vdots \\
1 & \bar{s}_{l_{m}}^{(N)} & \bar{c}_{l_{m}}^{(N) 1} & \ldots & \bar{c}_{l_{m}}^{(N) n} \\
0 & \left(\bar{s}_{l_{m}}^{(N)}\right)^{\prime} & \left(\bar{c}_{l_{m}}^{(N) 1}\right)^{\prime} & \ldots & \left(\bar{c}_{l_{m}}^{(N) n}\right)^{\prime} \\
1 & \bar{s}_{\Lambda}^{(N)} & \bar{c}_{\Lambda}^{(N) 1} & \ldots & \bar{c}_{\Lambda}^{(N) n}
\end{array}\right\}
$$

- For options with strike prices $X$ between $\left(K_{2 i-2}, K_{2 i-1}\right), i=1,2, \ldots$, when its upper bound is violated the arbitrage portfolio is given by

$$
\alpha_{i}=(-1)^{i}\left[-\bar{c}_{1}^{(N) X} B_{1 i}+\sum_{1}^{m}\left(\bar{c}_{l_{v}}^{(N) X} B_{2 v, i}-\left(\bar{c}_{l_{v}}^{(N) X}\right)^{\prime} B_{2 v+1, i}\right)\right] /|B|,
$$


$i=1, \ldots, n+2$, where $s_{l_{1}}, \ldots, s_{l_{m}}$ are determined by 1(a) in Proposition 6 and $B$ is given by

$$
\left\{\begin{array}{ccccc}
1 & \bar{s}_{1}^{(N)} & \bar{c}_{1}^{(N) 1} & \ldots & \bar{c}_{1}^{(N) n} \\
1 & \bar{s}_{l_{1}}^{(N)} & \bar{c}_{l_{1}}^{(N) 1} & \ldots & \bar{c}_{l_{1}}^{(N) n} \\
0 & \left(\bar{s}_{l_{1}}^{(N)}\right)^{\prime} & \left(\bar{c}_{l_{1}}^{(N) 1}\right)^{\prime} & \ldots & \left(\bar{c}_{l_{1}}^{(N) n}\right)^{\prime} \\
\vdots & \vdots & \vdots & \vdots & \vdots \\
1 & \bar{s}_{l_{m}}^{(N)} & \bar{c}_{l_{m}}^{(N) 1} & \ldots & \bar{c}_{l_{m}}^{(N) n} \\
0 & \left(\bar{s}_{l_{m}}^{(N)}\right)^{\prime} & \left(\bar{c}_{l_{m}}^{(N) 1}\right)^{\prime} & \ldots & \left(\bar{c}_{l_{m}}^{(N) n}\right)^{\prime}
\end{array}\right\}
$$

- For options with strike prices $X$ between $\left(K_{2 i-1}, K_{2 i}\right), i=1,2, \ldots$, when its lower bound is violated the arbitrage portfolio is given by (4); when its upper bound is violated the arbitrage portfolio is given by (2)

- Assume $n$ is even. Let $m=n / 2$.

- For options with strike prices $X$ between $\left(K_{2 i-2}, K_{2 i-1}\right), i=1,2, \ldots$, when its lower bound is violated the arbitrage portfolio is given by

$$
\alpha_{i}=(-1)^{i} \sum_{1}^{m+1}\left(\left(\bar{c}_{l_{v}}^{(N) X}\right)^{\prime} U_{2 v, i}-\bar{c}_{l_{v}}^{(N) X} U_{2 v-1, i}\right) /|U|,
$$
$i=1, \ldots, n+2$, where $s_{l_{1}}, \ldots, s_{l_{m}}$, are determined by $2(b)$ in Proposition 
6 and $U$ is given by

$$
\left\{\begin{array}{ccccc}
1 & \bar{s}_{l_{1}}^{(N)} & \bar{c}_{l_{1}}^{(N) 1} & \ldots & \bar{c}_{l_{1}}^{(N) n} \\
0 & \left(\bar{s}_{l_{1}}^{(N)}\right)^{\prime} & \left(\bar{c}_{l_{1}}^{(N) 1}\right)^{\prime} & \ldots & \left(\bar{c}_{l_{1}}^{(N) n}\right)^{\prime} \\
\vdots & \vdots & \vdots & \vdots & \vdots \\
1 & \bar{s}_{l_{m+1}}^{(N)} & \bar{c}_{l_{m+1}}^{(N) 1} & \ldots & \bar{c}_{l_{m+1}}^{(N) n} \\
0 & \left(\bar{s}_{l_{m+1}}^{(N)}\right)^{\prime} & \left(\bar{c}_{l_{m+1}}^{(N) 1}\right)^{\prime} & \ldots & \left(\bar{c}_{l_{m+1}}^{(N) n}\right)^{\prime}
\end{array}\right\}
$$

- For options with strike prices $X$ between $\left(K_{2 i-2}, K_{2 i-1}\right), i=1,2, \ldots$, when its upper bound is violated the arbitrage portfolio is given by $\alpha_{i}=(-1)^{i}\left[-\bar{c}_{1}^{(N) X} V_{1 i}+\sum_{1}^{m}\left(\bar{c}_{l_{v}}^{(N) X} V_{2 v, i}-\left(\bar{c}_{l_{v}}^{(N) X}\right)^{\prime} V_{2 v+1, i}\right)+\bar{c}_{\Lambda}^{(N) X} V_{n+2, i}\right] /|V|$,

$i=1, \ldots, n+2$, where $s_{l_{1}}, \ldots, s_{l_{m}}$, are determined by $2(a)$ in Proposition 6 and $V$ is given by

$$
\left\{\begin{array}{ccccc}
1 & \bar{s}_{1}^{(N)} & \bar{c}_{1}^{(N) 1} & \ldots & \bar{c}_{1}^{(N) n} \\
1 & \bar{s}_{l_{1}}^{(N)} & \bar{c}_{l_{1}}^{(N) 1} & \ldots & \bar{c}_{l_{1}}^{(N) n} \\
0 & \left(\bar{s}_{l_{1}}^{(N)}\right)^{\prime} & \left(\bar{c}_{l_{1}}^{(N) 1}\right)^{\prime} & \ldots & \left(\bar{c}_{l_{1}}^{(N) n}\right)^{\prime} \\
\vdots & \vdots & \vdots & \vdots & \vdots \\
1 & \bar{s}_{l_{m}}^{(N)} & \bar{c}_{l_{m}}^{(N) 1} & \ldots & \bar{c}_{l_{m}}^{(N) n} \\
0 & \left(\bar{s}_{l_{m}}^{(N)}\right)^{\prime} & \left(\bar{c}_{l_{m}}^{(N) 1}\right)^{\prime} & \ldots & \left(\bar{c}_{l_{m}}^{(N) n}\right)^{\prime} \\
1 & \bar{s}_{\Lambda}^{(N)} & \bar{c}_{\Lambda}^{(N) 1} & \ldots & \bar{c}_{\Lambda}^{(N) n}
\end{array}\right\}
$$

- For options with strike prices $X$ between $\left(K_{2 i-1}, K_{2 i}\right), i=1,2, \ldots$, when its lower bound is violated the arbitrage portfolio is given by (8); when its upper bound is violated the arbitrage portfolio is given by $(6)$. 
Proof: The proof is very similar to the case of second stochastic dominance. For brevity, it is omitted. For details, see Huang (2004b).

\section{Conclusions}

In this paper we derive $N$ th order stochastic dominance option bounds from concurrently expiring options. We show that given the prices of a unit bond, underlying stock, and $n$ option prices, the $k$ th order stochastic dominance option bounds are given by a pricing kernel the $(N-2)$ th derivative of which is $(n / 2)$-segmented and piecewise constant if $n$ is even or $((\mathrm{n}+1) / 2)$-segmented and piecewise constant if $n$ is odd. Since stochastic dominance rules are generally accepted, the derived option bounds in this paper are practically meaningful.

The results have important implications for arbitrage opportunities in the markets of concurrently expiring options. When the option bounds are violated we can construct arbitrage portfolios to take the advantage. 


\section{REFERENCES}

1. Arrow, Kenneth J. (1971). Essays in the Theory of Risk Bearing. Amsterdam: North Holland.

2. Basso, Antonella and Paolo Pianca (1997). "Decreasing Absolute Risk Aversion and Option Pricing Bounds." Management Science 43, 206-216.

3. Bernardo, Antonio E. and Olivier Ledoit. (2000). "Gain, Loss and Asset Pricing." Journal Political Economy 108, 144-172.

4. Bertsimas, Dimitris and Ioana Popescu (2002). "On the Relation between Option and Stock Prices: A Convex Optimization Approach." Operations Research 50, 358-374.

5. Black, Fischer and Myron Scholes (1973). "The Prices of Options and Corporate Liabilities." Journal of Political Economy 81, 637-654.

6. Boyle, P. and X. S. Lin (1997). "Bounds on Contingent Claims Based on Several Assets." Journal of Financial Economics 46, 383-400.

7. Brennan, Michael J. (1979). "The pricing of Contingent Claims in Discrete Time Models." Journal of Finance 34, 53-68.

8. Cochrane, John H. and Jesus Saa-Requejo (2000). "Beyond Arbitrage: Good-Deal Asset Price Bounds in Incomplete Markets." Journal Political Economy 108, 79-119.

9. Constantinides, G. M. and T. Zariphopoulou (1999). "Bounds on prices 
of contingent claims in an intertemporal economy with proportional transaction costs and general preferences." Finance and Stochastics 3, 345-369.

10. Constantinides, G. M. and T. Zariphopoulou (2001). "Bounds on derivative prices in an intertemporal setting with proportional transaction costs and multiple securities." Mathematical Finance 11 (3), 331-346.

11. Franke, G., R. C. Stapleton, and M. G. Subrahmanyam (1999). "When are Options Overpriced: The Black-Scholes Model and Alternative Characterizations of the Pricing Kernel." European Finance Review 3, 79-102.

12. Garman, M. (1976). "An Algebra for Evaluating Hedge Portfolios." Journal of Financial Economics 3, 403-428.

13. Grundy, Bruce D. (1991). "Option Prices and the Underlying Asset's Return Distribution." Journal of Finance 46, 1045-1070.

14. Huang, James (2004). "Option Pricing Bounds and the Elasticity of the Pricing Kernel." Review of Derivatives Research 7, 25-51.

15. Huang, James (2004a). "Risk Neutral Probabilities and Option Bounds: A Geometric Approach.” Lancaster University Management School working paper.

16. Huang, James (2004b). "Option Bounds and Second Order Arbitrage Opportunities." Lancaster University Management School working paper.

17. Huang, James (2004c). "DARA and DRRA Option Bounds from Concurrently Expiring Options." Lancaster University Management School 
working paper.

18. Huang, James (2004d). "Option Bounds from Concurrently Expiring Options When Relative Risk Aversion is Bounded." Lancaster University Management School working paper.

19. Huang, Chi-Fu and Robert H. Litzenberger Foundations for Financial Economics, New York: North-Holland 1988.

20. Jackwerth, Jens C. and Mark Rubinstein (1996). "Recovering Probability Distributions from Option Prices." Journal of Finance 51, 1611-1632.

21. Masson, Jean and Stylianos Perrakis (2000). "Option Bounds and the Pricing of Volatility Smile." Review of Derivative Research 4, 29-53.

22. Levy, Haim (1985). "Upper and lower Bounds of Put and Call Option Value: Stochastic Dominance Approach." Journal of Finance 40, 11971217.

23. Lo, A. (1987). "Semiparametric Upper Bounds for Option Prices and Expected Payoffs.” Journal of Financial Economics 19, 373-388.

24. Masson, Jean and Stylianos Perrakis (2000). "Option Bounds and the Pricing of Volatility Smile." Review of Derivative Research 4, 29-53.

25. Mathur, Kamlesh and Peter Ritchken (2000). "Minimum Option Prices Under Decreasing Absolute Risk Aversion." Review of Derivative Research $3,135-156$. 
26. Merton, Robert C. (1973). "Theory of Rational Option Pricing." Bell Journal of Economics and Management Science 4, 141-183.

27. Perrakis, Stylianos and Peter J. Ryan (1984). "Option Pricing Bounds in Discrete Time," Journal of Finance 39, 519-525.

28. Pratt, John W. (1964). "Risk Aversion in the Small and in the Large." Econometrica 32, 122-136.

29. Ritchken, Peter (1985). "On Option Pricing Bounds," Journal of Finance 40, 1219-1233.

30. Ritchken, Peter and Shyanjaw Kuo, (1989). "On Stochastic Dominance and Decreasing Absolute Risk Averse Option Pricing Bounds," Management Science 35, 51-59.

31. Rubinstein, Mark (1976). "The Valuation of Uncertain Income Streams and the Pricing of Options." Bell Journal of Economics 7, 407-425.

32. Ryan, Peter (2000). "A Tighter Option Bounds from Multiple Exercise Prices." Review of Derivatives Research 4, 155-87.

33. Ryan, Peter (2003). "Progressive Option Bounds from the Sequence of Concurrently Expiring Options." European Journal of Operations Research 151, 193-223.

34. Sachdeva, Kanwal (1986). "On the Equality of Two Lower Bounds on the Call Price: A Note.” Journal of Financial and Quantitative Analysis 21, 235-237. 
35. Stapleton, Richard C. and Marti G. Subrahmanyam (1990). "Risk Aversion and the Intertemporal Behavior of Asset Prices." Review of Financial Studies 3, 677-693. 